\title{
Les techniques de tissage ont-elles un sens? Un mode de lecture des tissus andins
}

Are weaving techniques one way street?Are Weaving Techniques One Way

Street? A Way of Looking at Andean Textiles

\section{Sophie Desrosiers}

\section{(2) OpenEdition}

Journals

Édition électronique

URL : https://journals.openedition.org/tc/5002

DOI : $10.4000 /$ tc. 5002

ISSN : 1952-420X

Éditeur

Éditions de l'EHESS

Édition imprimée

Date de publication : 30 juin 2010

Pagination : 263-285

ISSN : 0248-6016

Référence électronique

Sophie Desrosiers, «Les techniques de tissage ont-elles un sens ? Un mode de lecture des tissus andins », Techniques \& Culture [En ligne], 54-55 | 2010, mis en ligne le 30 janvier 2013, consulté le 29 septembre 2022. URL : http://journals.openedition.org/tc/5002 ; DOI : https://doi.org/10.4000/tc. 5002 


\section{LES TECHNIQUES DE TISSAGE ONT-ELLES UN SENS ?}

\section{Un mode de lecture des tissus andins}

in Techniques et culture 12, $1988: 21-56$

L'importance des textiles dans les Andes n'est plus à démontrer ${ }^{1}$. Des auteurs ayant plus directement étudié des étoffes préhispaniques ont déjà souligné l'intérêt que présente ce domaine pour la connaissance des sociétés andines (Gayton 1961, Bird 1985). C'est ce qu’a confirmé John V. Murra dont les recherches ethnohistoriques ont révélé les diverses fonctions -économique, sociale, politique, militaire, magique...- dévolues aux étoffes incas (Murra 1962).

Malgré leur abondance et leur diversité, ces sources sont cependant incomplètes et de valeur inégale. Au contraire de ce qui s'est produit sur la côte, le climat humide des hautes terres n'a pas permis la conservation des textiles préhispaniques. Il en résulte que la production ancienne de la zone montagneuse est presque uniquement connue à travers des pièces trouvées dans le désert côtier et attribuables aux cultures dont l'expansion culturelle et éventuellement territoriale a atteint l'Océan Pacifique - avant tout les cultures Chavin, Tiahuanaco, Huari et Inca. Au cours de la Période Coloniale, les rites funéraires ayant été bouleversés par les Espagnols, les textiles ne sont plus conservés en abondance dans les tombes et les pièces de cette période sont en nombre réduit par rapport aux exemples préhispaniques. Enfin aujourd'hui, les traditions textiles ont peu survécu sur la côte. À ces disparités régionales et chronologiques, il faut ajouter que les collections conservées dans de nombreux musées sont dépourvues de contexte archéologique, et bien souvent d'information sur leur provenance, qu'elles aient été constituées à la fin du XIX ${ }^{e}$ siècle quand les archéologues n'employaient pas encore de méthodes rigoureuses, ou qu'elles proviennent du marché international longtemps alimenté par les trouvailles des pilleurs de tombes. 


\section{Figure 1}

Andes centrales et centre-sud grandes divisions culturelles (d'après L. G. Lumbreras, $1974: 2$; Rivera, 1984 : figure 2).
En dépit de ces handicaps, les travaux publiés depuis un peu plus d'un siècle ont conduit à reconstituer des pans importants de l'histoire des textiles des Andes. Sur le plan des techniques, l'étude comparative de ces résultats a mis en évidence des phénomènes de diffusion, d'innovation et de blocage qui se sont produits dans des régions et à des époques diverses pendant la période préhispanique (Conklin 1970, 1975, Wallace 1979, Frame 1982, Bird 1985, Grieder 1986). Elle a souligné certains changements apportés par les Espagnols, en particulier l'emploi de nouveaux matériaux comme la laine de mouton, la soie, les fils d'or ou encore l'introduction de

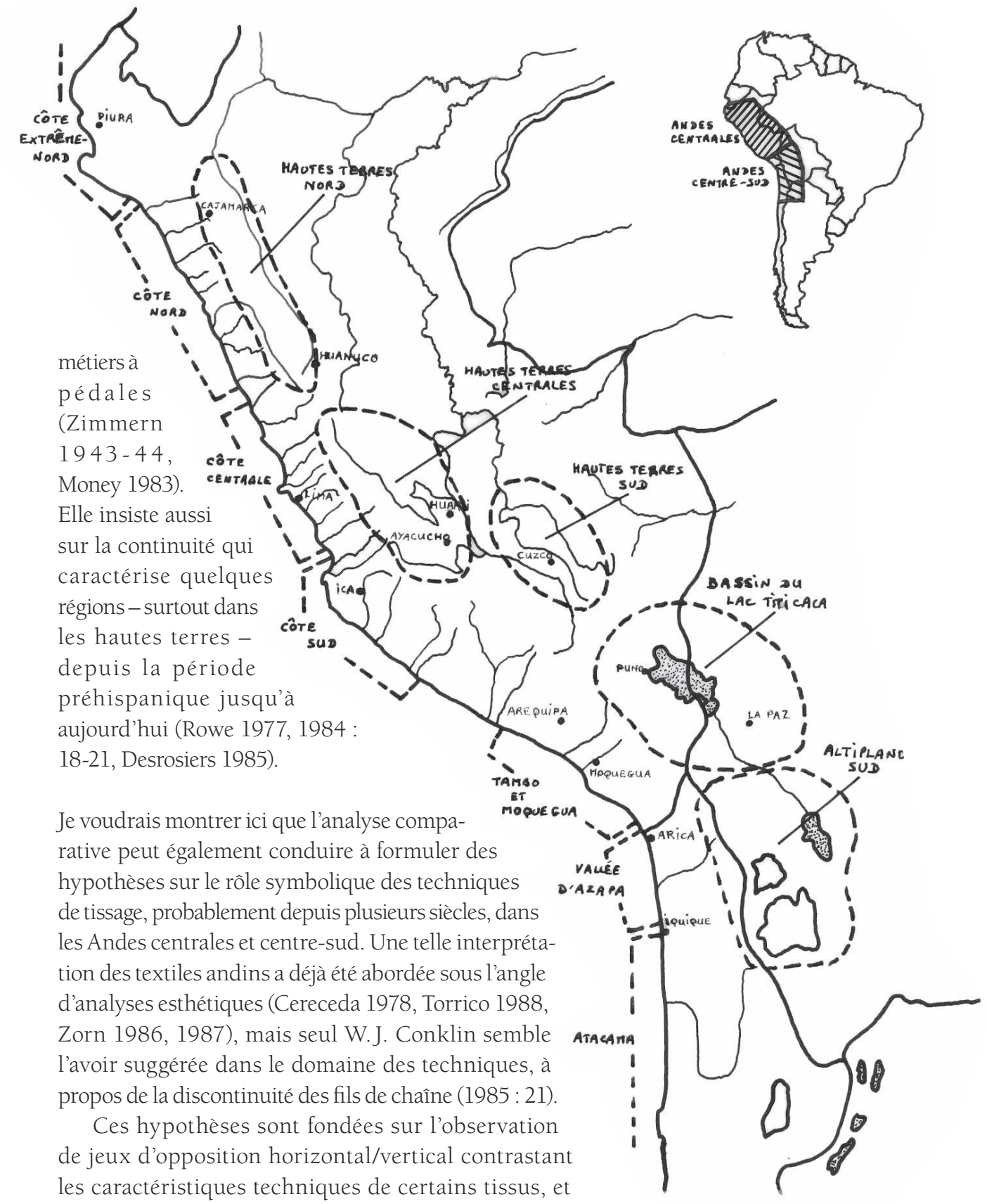




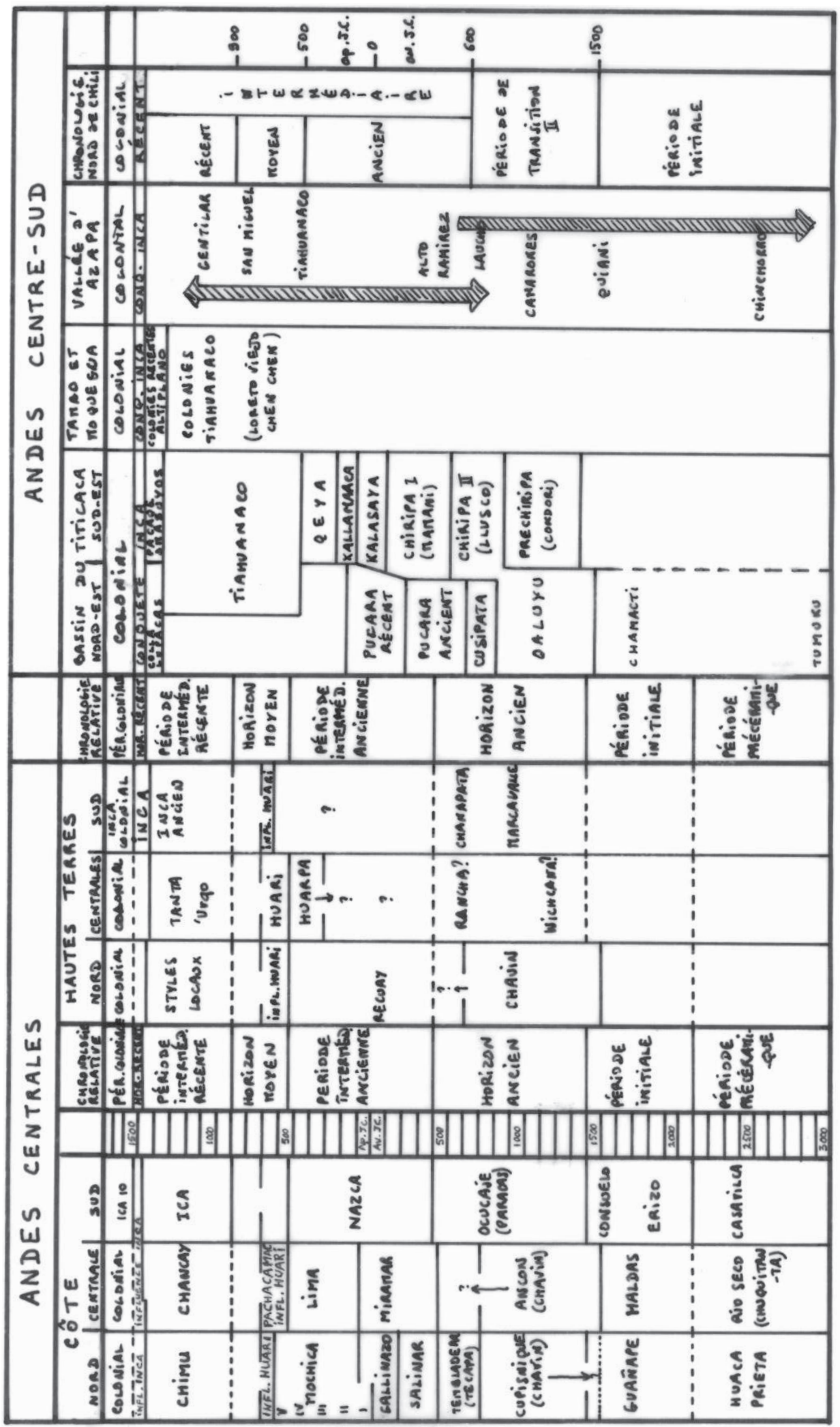

Tableau 1

Chronologie des Andes centrales et centre-sud (d'après A. P. Rowe et

Menzel, 1982 : introduction, Rivera, 1984 : figure 1). 


\section{Figure 2} d'Harcourt, 1934 : 15, figure 3).
Métier à tisser andin (d'après

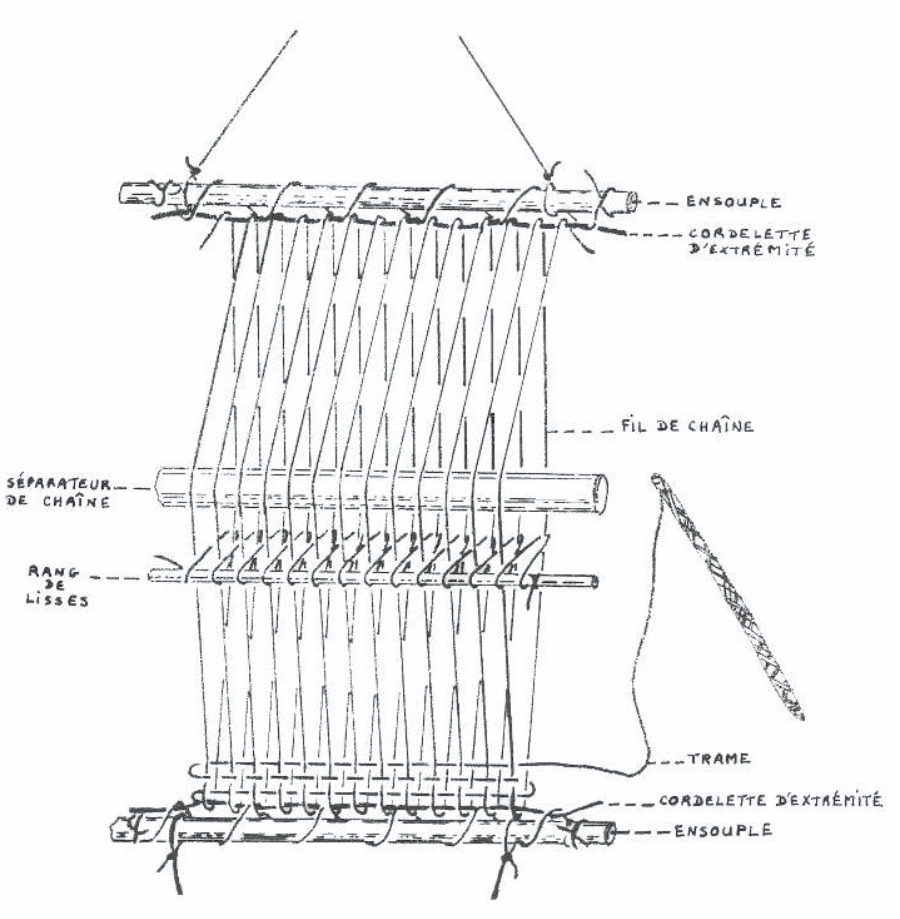

Figure 3

Trois exemples de structures permettant de créer un décor : (a) avec les fils de chaine et de trame, (b) avec les seuls fils de chaine, (c) avec les seuls fils de trame. sur la correspondance de ces distinctions avec une division de l'espace et une opposition féminin/masculin qui sont opérationnelles pour l'étude de formes de pensée andines très diverses (Zuidema 1964, Platt 1976, Harris 1978). Des rapports entre genre et tissage ont déjà été notés dans d'autres régions du monde - par exemple en Afrique du Nord (Messick 1987 : 213) et en Asie du Sud et du Sud-Est (March 1983, Niessen 1985)-, mais aucune étude ne les a jusqu'ici mis en évidence dans les Andes.

Après avoir rappelé quelques éléments techniques de la fabrication et de l'utilisation des tissus dans cette aire culturelle, j'examinerai donc successivement comment les oppositions techniques repérées correspondent d'une part à une division de l'espace en deux grandes régions géographiques et d'autre part, localement, au contraste entre les catégories du féminin et du masculin.

\section{Fabrication et utilisation des tissus comme vêtement dans les Andes centrales et centre-sud}

Le métier à tisser de tradition andine est extrêmement simple (figure 2). Il comporte deux barres - ou ensouples - entre lesquelles sont tendus les fils de chaine. Ces derniers sont maintenus solidaires du métier par l'intermédiaire de cordelettes d'extrémité qui passent dans les boucles des fils et sont directement attachées aux ensouples à l'aide d'un fil très résistant ${ }^{4}$.

La tension est variable, et contrôlée par le tisserand, dans le cas des métiers à la ceinture $^{5}$. Dans les autres cas où les ensouples sont attachées à des supports, la tension est fixe et la chaîne diversement orientée - horizontalement, obliquement ou verticalement - selon la disposition de ces supports ${ }^{6}$. 
Un ou parfois plusieurs rangs de lisses et une barre d'écartement permettent au tisserand de séparer les fils de chaîne en deux nappes entre lesquelles il introduit transversalement la trame. Les structures - ou entrecroisements des fils de chaîne et de trame - sont extrêmement diverses et souvent employées pour créer un décor au cours du tissage. Elles se divisent alors en trois catégories : les structures dans lesquelles la chaîne et la trame ont un même rôle dans l'élaboration des motifs, celles où les dessins sont obtenus par les seuls fils de chaîne et enfin à l'inverse, celles où ce rôle incombe aux trames (figure 3).

Pour libérer l'étoffe du métier, trois méthodes sont plus généralement employées. Avec la première, qui est la plus fréquente, la pièce est tissée sur toute la longueur de la chaîne, puis détachée du métier en retirant les fils qui maintiennent les cordelettes d'extrémité aux ensouples. La seconde consiste à couper l'extrémité non tissée des fils de chaine et à rentrer ceux-ci à l'aiguille dans l'étoffe, enfin la troisième, à transformer les deux extrémités non tissées en franges. Avec les deux premières méthodes, les tissus tombés du métier possèdent quatre lisières et avec la troisième des franges remplacent les lisières transversales.

À part certaines exceptions, ces pièces de tissu finies, que j'appellerai désormais lés, sont de dimensions réduites. En particulier quand elles ont été tissées sur un métier à la ceinture, leur largeur est habituellement limitée par l'envergure des bras du tisserand et ne dépasse pas $75-80 \mathrm{~cm}$. Ces lés ne sont pas coupés mais employés tels quels ou bien après avoir été pliés, cousus et éventuellement assemblés avec d'autres afin d'obtenir les dimensions et les formes recherchées ${ }^{7}$.

Une partie importante des textiles préhispaniques retrouvés dans les tombes sont des pièces de vêtement et beaucoup de tissus de facture traditionnelle trouvent aujourd'hui une telle utilisation. Leurs types varient selon les époques, le sexe du porteur et son origine géographique ; cependant certaines connaissent une vaste distribution. C'est le cas plus particulièrement des tuniques masculines - ou uncu ${ }^{8}$ - encore portées dans certaines communautés des hautes terres boliviennes comme Chipaya (Carangas) (Métraux 193435) et dont l'exemple le plus ancien qui soit connu remonte à l'Horizon Ancien (Kajitani 1982 : pl. 7). De forme généralement carrée ou rectangulaire, elles se composent d'un nombre limité de lés - très souvent un ou deux -tissés, pliés et cousus de manière à laisser des fentes verticales pour passer la tête et les bras (figure $4 \mathrm{a}, 4 \mathrm{~b}$ et 5); des manches ont parfois été exécutées avec le corps ou ajoutées ensuite. Dans de nombreuses régions, cette tunique a été remplacée par le poncho, une pièce de

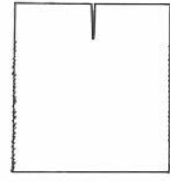
a

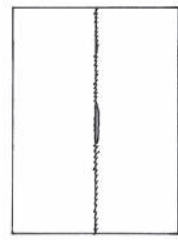

c

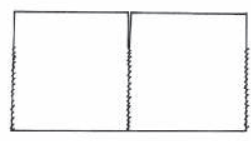

b

Figure 4 Constructions les plus courantes des tuniques masculines sans manches et des ponchos : (a) tuniques avec un seul lé, (b) tuniques avec deux lés, (c) poncho fait de deux lés. construction semblable mais non cousue sous les bras (figure 4c) ${ }^{9}$. Le vêtement masculin comporte d'autres pièces et accessoires mais il n'est pas question d'en faire aujourd'hui une étude comparative car ils sont difficiles à identifier, leur distribution est plus limitée et, contrairement aux tuniques, il existe peu d'études les concernant.

Le vêtement féminin est beaucoup moins bien connu, au moins pour la période préhispanique, car la plus grande partie des pièces exhumées des tombes sont masculines et l'iconographie céramique est imprécise et tout aussi limitée (Montell 1929 : 93-95, 110-111, 126, 164). À partir du xve siècle, il est possible de se faire une idée de sa composition grâce 


\section{Figure 5}

Dessins de Guaman Poma de Ayala : (a) I'Inca Virococha vêtu d'une tunique décorée de tocapus - unités décoratives de forme carrée ou rectangulaire disposées de manière répétitive -,

et (b) un capitaine de Colla Suyu, quart sud de l'Empire Inca, avec sa tunique rayée (Guaman Poma, $1936: 106$ et 109).

Figure 6

Dessins de Guaman Poma de Ayala (représentant des Coya ou femmes d'Inca vêtues d'une robe - acsu - et d'un châle - lliclla: (a) pour la huitième Coya, Mama lunto Cayan, ces deux pièces sont décorées de tokapus,

(b) pour la deuxième, Mama Ocllo, elles sont de simples rayures (Guaman Poma, $1936: 134$ et 138). aux quelques pièces incas retrouvées, aux écrits espagnols, à l'iconographie et aux pièces plus nombreuses de la Période Coloniale ainsi qu'au vêtement féminin actuel. Dans les hautes terres, l'élément principal est une robe - ou acsu -, grand rectangle d'étoffe enroulé autour du corps, maintenu aux épaules par deux fibules - les tupu - et à la taille par une ceinture - ou chumpi (figure 6) (Montell 1929 : 226-229). Aujourd'hui cette pièce est sou-
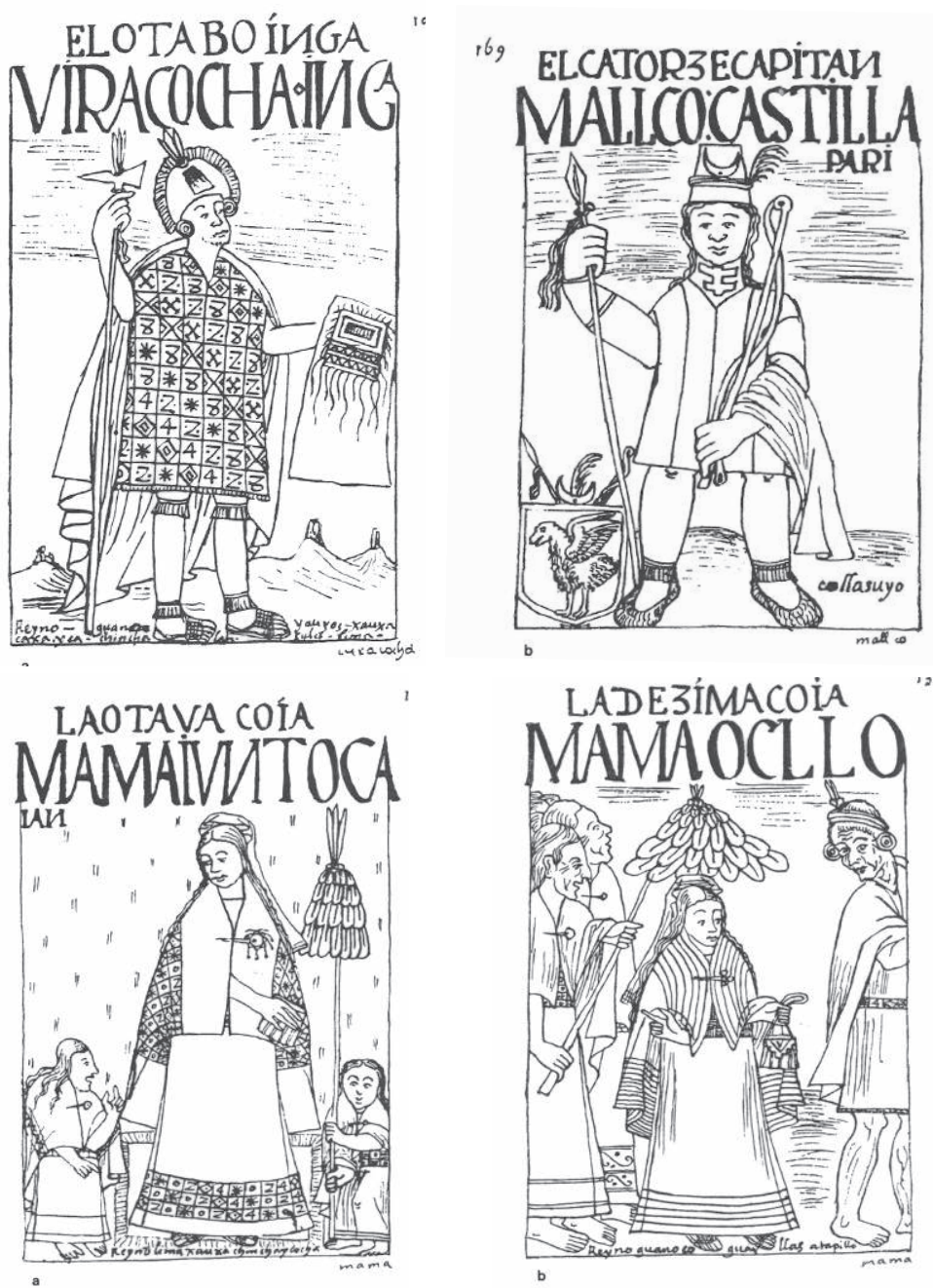

vent de plus petite dimension et réduite au rôle de dessus de robe ou même de jupe (Gisbert, Arze y Cajias 1987 : 65-67). Ces différentes versions sont accompagnées d'un châle - ou lliclla - de forme carrée ou rectangulaire qui couvre les épaules et le haut du corps; il se trouve maintenu en place par une fibule ou un noeud sur la poitrine. Les exemples jusqu'ici repérés montrent que robes ou dessus de robe et châles se composent aussi de quelques lés - un seul ou plus souvent deux - cousus le long d'une lisière longitudinale, tandis que les jupes sont faites d'un seul lé plus long (figure 7). Les femmes portent aussi d'autres pièces et accessoires mais pour les mêmes raisons que celles données plus haut dans le cas des hommes, ils ne peuvent être pris en compte ici. 
Cette trop courte présentation montre que les pièces principales des vêtements masculin et féminin sont habituellement construites d'une manière standardisée sur l'ensemble de la zone considérée. Il suffit généralement d’un lé de forme carrée ou rectangulaire, ou bien de deux lés assemblés, éventuellement pliés. L’absence de coupe implique que le tisserand exécute ces lés et donc ourdisse les fils de chaîne longitudinalement sur le métier dans les dimensions requises par la pièce en projet. Cependant, comme le met en évidence le nombre élevé de travaux de synthèse publiés sur les tuniques masculines de plusieurs périodes et sur la répartition des structures ou entrecroisements des fils, des différences apparaissent quand on entre dans les détails. En particulier, le sens suivant lequel les tisserands ont exécuté les lés pour fabriquer tuniques et ponchos varie selon deux régions géographiques.

\section{Répartition géographique des tuniques et ponchos masculins et opposition horizontal/vertical}

Les tuniques et ponchos masculins s'opposent selon l'orientation horizontale ou verticale des fils de chaîne ou de trame, cela dépend des structures employées pour les tisser. Tout d'abord, je considérerai les tuniques exécutées en tapisserie - une toile dans laquelle la trame cache entièrement la chaine et crée un décor par des changements de couleur dans la largeur de l'étoffe. Puis j'examinerai les tuniques et ponchos tissés avec des structures autres que la tapisserie mais formant aussi un décor par la trame ou bien avec des structures conduisant à créer des motifs avec les fils de chaîne.

\section{Les tuniques en tapisserie}

Développée depuis l'Horizon Ancien, la tapisserie s'est ensuite généralisée dans toutes les Andes Centrales et Centre-Sud (Conklin, 1975, Ulloa 1981a). De nombreuses publications portent sur des tuniques tissées avec cette structure de Tiahuanaco à la fin de la Période Coloniale.

Dès le début du siècle, sans tenir compte de la fonction précise des pièces ou des fragments qu'il étudiait, Max Schmidt notait une différence entre les tapisseries Huari et celles, postérieures, des côtes septentrionale et centrale du Pérou (Schmidt 1910 : 154-156 et 1911 : 3-5). Ces dernières étaient principalement à relais, c'est-à-dire avec des fentes verticales créées par le non-crochetage des trames aux changements de couleur, tandis que les premières avaient des liures ou crochetages simples aux changements de couleurs verticaux. Les travaux postérieurs confirmèrent et précisèrent ces observations en montrant toutefois que cette différence technique n'indiquait pas 


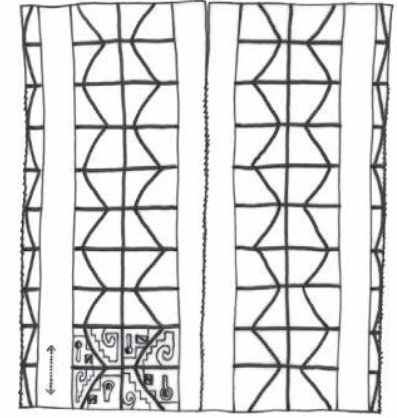

10a

a

un écart chronologique - comme le pensait Schmidt - mais une distinction entre les cultures de la côte et celles des hautes terres.

Dans le cas des tuniques masculines, plusieurs travaux constatent l'extrême standardisation de leur construction au sein des cultures des hautes terres. Ils relèvent la différence des types de tapisserie et ajoutent d'autres caractéristiques qui distinguent les pièces des hautes terres de celles de la côte. J'en retiendrai deux. La première est la présence de lisières longitudinales renforcées - c'est-à-dire comportant des fils doubles ou triples ou seulement plus gros - et la seconde consiste dans un changement de la direction des fils de chaîne qui, de longitudinaux sur le métier employé par le tisserand, deviennent horizontaux dans les pièces finies.

Les tuniques en tapisserie attribuées aux hautes terres - Tiahuanaco, Huari, incas et de la Période Coloniale - présentent comme caractéristiques communes d'avoir été tissées en tapisserie avec des liures simples aux changements de couleur

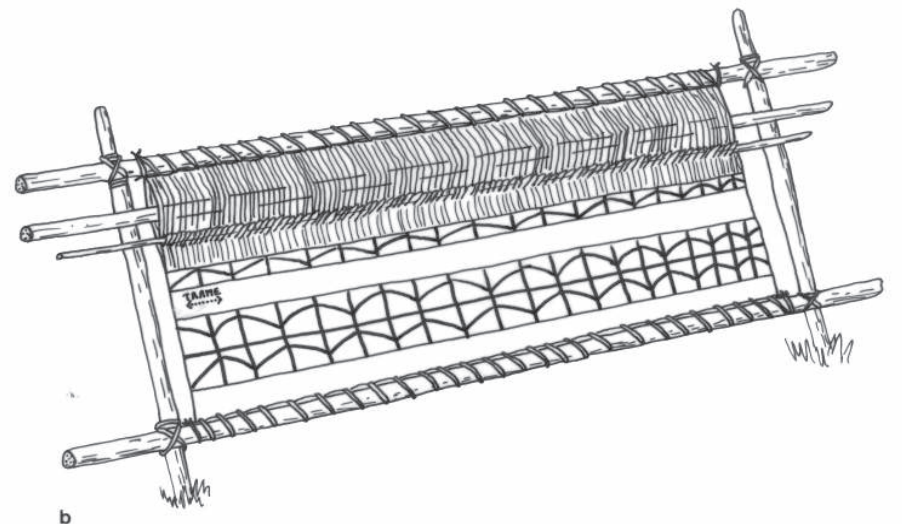
verticaux, de comporter généralement des lisières longitudinales renforcées, et d'être construites avec la chaîne orientée horizontalement, à l'inverse de la position de l'étoffe devant le tisserand au cours de sa fabrication. Les tuniques Tiahuanaco, incas et coloniales forment un sousgroupe construit avec un seul lé tandis que celles attribuées à la culture Huari en comprennent deux (figure 8a) ${ }^{10}$. Les dimensions relativement standardisées pour chaque culture montrent que les lés ont été tissés beaucoup plus larges que hauts -par exemple 1,85 $\mathrm{m}$ de large par 0,76 $\mathrm{m}$ de haut pour les tuniques incas à un seul lé ou bien $2 \mathrm{~m}$ de large par 0,56 $\mathrm{m}$ de haut pour celles, Huari, faites de deux lés (figure 8b) (Sawyer 1963: 27, Rowe $1978: 7$ ).

Les tuniques en tapisserie tissées sur la côte ont été moins

Figure 8 Tunique masculine en tapisserie des hautes terres composée de deux lés.

Un exemple Huari

(coll. A. C. Glassel Jr., Houston) :

(a) tunique,

(b) un lé sur le métier à tisser (d'après la reconstruction de Bird et Skinner 1974 : figure 5 replubiée par Conklin, 1986 : figure 4). étudiées mais elles sont reconnues comme ayant des traits techniques opposés à celles des hautes terres (Rowe $1978: 8$ et $1986:$ 153). En particulier, les pièces Chancay et Chimu des côtes centrale et nord du Pérou sont généralement en tapisserie à relais, avec des lisières longitudinales qui n'ont pas été jusqu'ici décrites comme renforcées, et sont construites avec la chaîne orientée verticalement, comme elle apparaissait au tisserand sur le métier. Ces tuniques sont habituellement construites avec deux lés plus longs que larges si l'on exclut les manches éventuelles (figure 9a) (Rowe 1984).

La rareté relative des travaux sur les tuniques en tapisserie de la côte et la discontinuité des séries de tuniques attribuables aux hautes terres ne permettent de reconstituer qu'une partie du tableau initial. Quelques exceptions, peut-être tissées pendant l'Horizon Récent sur la côte extrême-sud du Pérou - aujourd'hui rebaptisée région de Tarnbo et Moquegua - manifestent une très forte similarité technique avec les tuniques incas (Rowe 1978 : 24) et font douter de la simplicité ou plutôt de la rigidité du modèle « hautes terres/côte » dont la définition géographique a probablement connu une évolution au cours du temps. De plus, il est possible que les tuniques masculines Pucarâ et Recuay aient été construites sur le même modèle que celles des cultures postérieures des hautes terres. D'après les quelques fragments d'étoffes sur lesquels j’ai trouvé des analyses précises, les tisserands Pucarâ et Recuay auraient employé le même type de tapisserie à liures simples et, pour les fragments attribués aux seconds, le décor indique sans ambiguité que les tissus étaient employés avec la chaîne horizontale ${ }^{11}$. Mais seule l'identification de tuniques ayant les caractéristiques techniques retenues permettra d'ajouter ces deux cultures 
au groupe défini plus haut et de dater du début de la Période Intermédiaire Ancienne la possible naissance de l'opposition entre pièces de la côte et pièces des hautes terres.

Sur le plan de l'interprétation, ces divers contrastes peuvent être attribués tout simplement à des différences importantes dans les types de métiers employés. Un métier à tisser à la ceinture sur la côte expliquerait la présence de lés de largeur limitée dans les tuniques de cette région (figure 9b). Un métier fixe, vertical ou oblique comme celui qu'ont reconstruit J. B. Bird et M. D. Skinner pour une tunique Huari (figure 8b) faciliterait le tissage de lés de hauteur plus limitée que la largeur et rendrait peut-être nécessaire le renforcement des lisières longitudinales ${ }^{12}$. Cependant, comme les tuniques et ponchos tissés avec d'autres structures que la tapisserie s'opposent aussi selon l'orientation horizontale ou verticale de certains fils et respectent une répartition géographique assez semblable, on peut s'interroger sur l'interprétation uniquement technique de cette première observation.

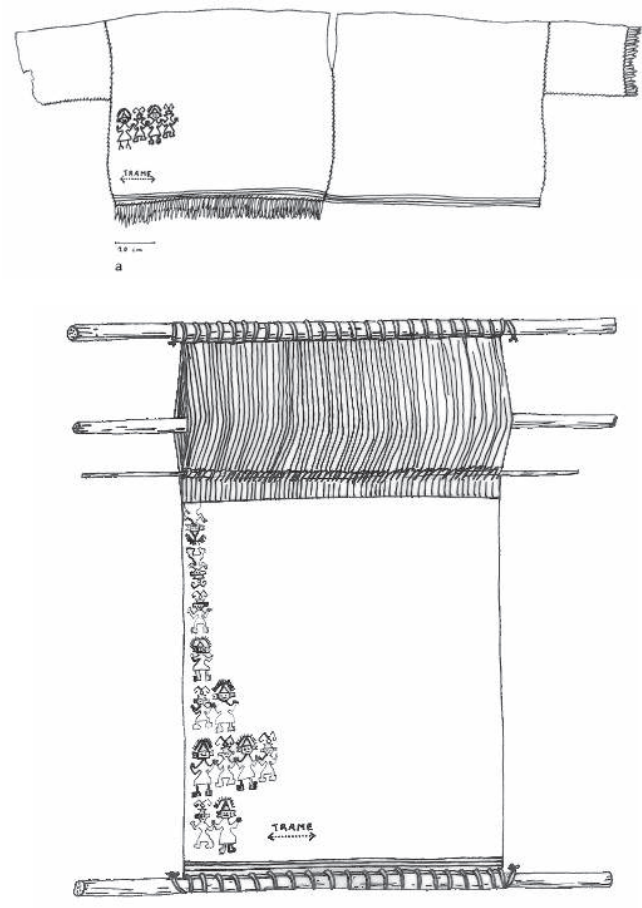

\section{Figure 9 Tunique masculine en tapisserie de la côte composée de deux lés avec des manches.}

Un exemple Chimu (T. M. W. $=1961.3$. d’après A.P. Rowe 1984 : pl. 12) ; (a) tunique,

(b) un lé sur le métier à tisser (reconstruction).

On aurait le même tracé pour une tunique de la côte à décor pour la trame autre que la tapisserie comme A.P. Rowe, 1984 : pl.7).

\section{Les tuniques et ponchos tissés avec des structures autres que la tapisserie}

Dans son ouvrage sur les tissus anciens du Pérou, Raoul d'Harcourt (1934 : 120) avait remarqué que les structures permettant de créer un dessin par la chaîne trouvaient souvent leur équivalent parmi celles qui conduisaient à faire un décor en trame selon ce qu'il a nommé « ...le principe d'interchangeabilité du rôle de la trame et de la chaîne... ». Or des travaux plus récents de A. P. Rowe et L. Ulloa ont montré que ces deux groupes de structures se répartissent en deux régions géographiques distinctes.

D’après Rowe (1977 : 113-114), dont l'étude se limite aux Andes centrales, les structures permettant de créer un décor par la chaîne sont probablement originaires des hautes terres. Elles apparurent sur la côte à la fin de la Période Intermédiaire Ancienne mais jouèrent jusqu'à aujourd'hui un rôle beaucoup plus important dans les hautes terres que sur la côte. Quant aux structures créant un décor par la trame, à l'exception de la tapisserie commune aux deux régions, elles ont eu une importance bien plus grande sur la côte. La seule entorse au modèle " côte/hautes terres » est à nouveau la région de Tambo et Moquegua où pendant l'Horizon Récent les tisserands employèrent fréquemment des structures à dominante chaîne. Les travaux de L. Ulloa (1981a : 99) ont pour leur part souligné l'apparition de structures à dominante chaîne dans la vallée d'Azapa sur la côte nord des Andes centre-sud, à partir des phases El Laucho et Faldas del Morro, à la fin de l'Horizon Ancien. Cette précision conduit à modifier l'opposition précédente en « hautes terres et vallée d'Azapa/côte moins vallée d'Azapa », avec la réserve évoquée plus 


\section{Figure 10}

Tunique masculine des hautes terres au décor de rayures réalisé par la chaîne, composée d'un seul lé.

Un exemple colonial de Bolivie en toile à dominante chaîne rayée (Adelson et Tracht $\left.1983: n^{\circ} 1\right)$. Noter la ressemblance avec la tunique de la figure $5 b$.

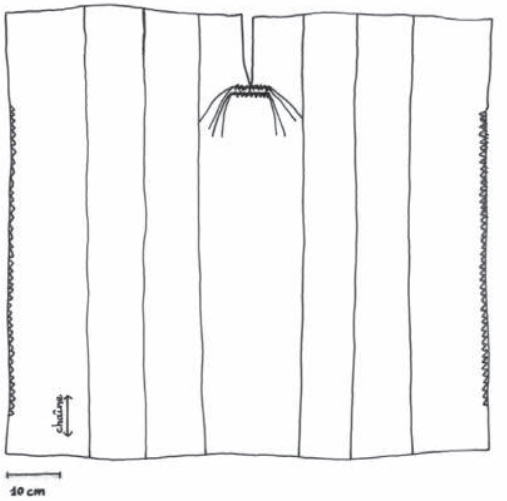

haut pour Tambo et Moquegua, ou bien à faire l'hypothèse que les Andes centre-sud n'entrent pas dans ce schéma. Pour ne pas alourdir l'exposé je continuerai toutefois à nommer comme précédemment les deux régions en opposition.

Les tuniques et les ponchos tissés avec ces structures sont généralement portés avec la chaîne verticale, qu'ils soient préhispaniques, coloniaux ou plus récents ${ }^{13}$. Leurs décors sont donc créés par des fils orientés horizontalement sur la côte et verticalement dans les hautes terres (figures 9 et 10).

Cette observation semble contredire la

précédente, puisque la chaîne des tuniques en tapisserie est orientée horizontalement dans les hautes terres et verticalement sur la côte. Mais si l'on accorde davantage d'importance aux fils apparaissant à la surface de l'étoffe, c'est à la direction de la trame, élément dominant des tapisseries, qu'il faut s'en tenir. Or celle-ci est bien horizontale dans les tuniques masculines de la côte et verticale dans celles des hautes terres. Un tel renversement de point de vue peut sembler une gymnastique gratuite, mais deux autres observations renforcent cette position.

La première est la ressemblance que deux auteurs au moins ont notée entre les rayures de tuniques en tapisserie des hautes terres et celles d'autres uncu contemporains au décor créé par les fils de chaîne : les rayures étaient de même couleur et suivaient plus ou moins le même rythme dans des exemples Tiahuanaco (Oakland 1986 : 107) et d'autres de la fin de l'Empire Huari (Rowe 1986: 161) ${ }^{14}$.

La deuxième observation concerne un point plus technique. Parmi les caractéristiques qui différencient les tuniques en tapisserie de la côte de celles des hautes terres, on a noté que les lisières longitudinales des lés composant les pièces Tiahuanaco, Huari, incas et probablement coloniales sont renforcées. Or après examen de nombreuses pièces au décor obtenu par la chaîne, les tuniques et ponchos présentant des lisières transversales y gardent normalement les cordelettes d'extrémité, ce qui prowduit le même aspect qu'une lisière renforcée. De cette manière, quelle que soit la structure employée, ces tuniques des « hautes terres » ont non seulement des motifs obtenus avec les fils orientés dans la même direction mais aussi généralement un renforcement situé à une place identique, dans leur partie inférieure.

Un rapprochement s'impose, dans les « hautes terres », entre les uncu en tapisserie et les tuniques et ponchos au décor construit avec les fils de chaîne. Sur la côte, on observe de même des ressemblances entre les décors des tuniques en tapisserie et ceux qui sont également créés par la trame mais avec d'autres structures (par exemple Rowe 1984 : pl. 3,7 ou fig. 132 et 133). Finalement, on est surpris de rencontrer une certaine homogénéité à l'intérieur des deux groupes de pièces malgré les structures différentes avec lesquelles elles ont été tissées. L'opposition entre les uncu de la côte et les tuniques et ponchos des hautes terres devient plus nette bien qu'un certain nombre de contre-exemples soient apparus au cours des recherches. Une tunique de la côte centrale, attribuée à la Période Intermédiaire Récente, a été tissée avec des structures à dominante trame et construite avec la trame verticale (Rowe 1977 : fig. 100), et les ponchos de Tarabuco (Chuquisaca, Bolivie), en toile à dominante chaîne 
rayée, sont toujours portés avec la chaîne horizontale (Meisch 1986 : figures 4-10) ; d'après Valeriano Thola (communication personnelle, août 1988), il en serait de même à Calcha et à Yura (potosi Bolivie).

Quelle est la signification de ces exceptions? Une inversion ? Le résultat de déplacements de population ou d'une influence d'une région sur une autre? Un abandon de comportements antérieurs? La localisation bien précise des quelques cas ethnographiques relevés et l'uniformité du renversement du sens dans lequel sont portées ces pièces a probablement une signification que seules des enquêtes de terrain permettront de comprendre. Cependant, ces contre-exemples ne semblent pas remettre fondamentalement en question une hypothèse qui, par la récurrence de ses probables manifestations, conduit à penser que les caractéristiques techniques soulignées signifient davantage que des différences d'outils et de savoir-faire. C'est ce que montrent les exemples suivants, qui utilisent la même opposition pour exprimer localement des distinctions entre pièces de vêtement féminin et masculin.

\section{Distinctions locales entre pièces de vêtement féminin et masculin et opposition horizontal/vertical}

Les divers exemples de distinction entre pièces de vêtement féminin et masculin fondés sur l'orientation horizontale ou verticale de certains traits techniques se situent dans trois régions différentes : les hautes terres, la côte, et également le piémont amazonien.

\section{Les hautes terres}

Les traditions textiles encore très vivantes des hauts plateaux boliviens fournissent d'abondants éléments de réflexion. Dans une région où tuniques masculines, ponchos, robes, dessus de robe, jupes et châles sont aujourd'hui presque toujours décorés par la chaîne, les femmes portent la robe et ses variantes de telle manière que les fils formant le décor sont orientés horizontalement ${ }^{15}$ (figure 11) à l'opposé des hommes qui, comme on l'a vu précédemment, revêtent le plus souvent tuniques ou ponchos avec la chaîne verticale (figure 10). En ce qui concerne le châle, les femmes semblent le porter aujourd'hui bien souvent avec ces mêmes fils horizontaux mais aussi parfois verticaux ${ }^{16}$. Toutefois les exemples que j'ai rencontrés dans l'iconographie coloniale et du xix ${ }^{e}$ siècle montrent jusqu'ici sans exception qu'elles revêtaient alors le châle, comme la robe, avec les rayures et donc probablement les fils de chaîne horizontaux ${ }^{17}$.

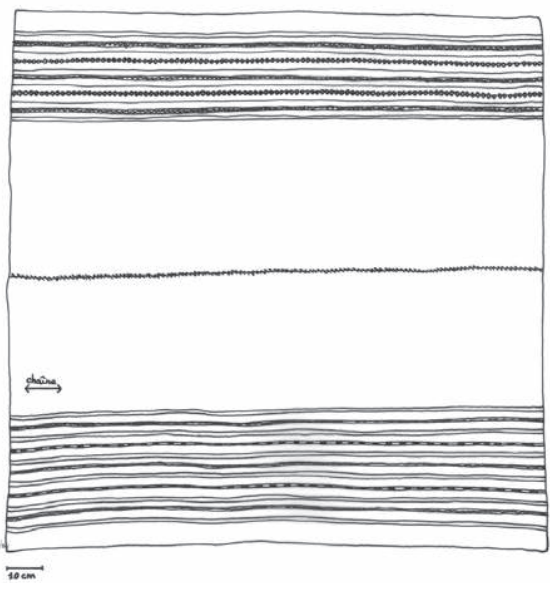

\section{Figure 11}

Robe des hautes terres tissée avec des structures créant un décor par la chaîne et composée de deux lés.

Un exemple colonial de Bolivie (Adelson and Tracht 1983: n 36). Noter la ressemblance avec la robe de la figure $6 b$. 
Des robes et des châles au décor créé par la chaîne se trouvent parmi les étoffes découvertes à Pachacamac dans le cimetière des femmes sacrifiées par les Incas (Uhle 1903 : 90-92). Uhle explique que ces châles ont des rayures en travers - crosswise - sauf deux qui les ont en longueur - lengthwise - mais il ne donne aucune explication sur ce qui lui a permis d'orienter ces pièces qu'il décrit comme carrées. Par sa forme nettement rectangulaire, le châle de style inca dont A.P Rowe a publié une photo se trouve avoir la chaîne verticale (Rowe 1978 : fig : 81). Enfin, une statuette inca découverte à Cerro del Plomo au Chili et revêtue de toute évidence d'une robe et d'un châle de ce type est le seul exemple montrant pour le moment comment ces pièces étaient alors portées (Lothrop 1979 : 226) : elles ont toutes deux la chaîne horizontale. Ces exemples et contre-exemples font penser à nouveau que le châle reste étranger à cette opposition féminin/masculin. C'est peut-être ce que veut dire Bertonio, au début du XVIII siècle, quand il mentionne cette opposition dans la définition du succullu, rite de passage des enfants aymaras (Bertonio 1984 : 323). Pour la petite fille, il ne fait état que de la partie basse du corps. À propos de ce texte, Thérèse Bouysse-Cassagne écrit :

« [...] la seconde phase [du rite] assigne à l'enfant un rôle différent selon qu'il est du sexe masculin ou féminin. En effet, on revêt le garçon d'une chemise traversée verticalement de trois fils de couleur : le sucullu cahua couvre le haut du corps. La fillette reçoit une jupe (huampaña) ornée d'une bande tissée horizontalement, qui couvre la partie basse du corps. Ce qui appartient aux hommes est haut et vertical, ce qui appartient aux femmes est bas et horizontal » (souligné par S. D.) (1978: $1069 ; 1987: 237)$.

Figure 12

Châle des hautes terres tissé en tapisserie et composé d'un seul lé. Un exemple colonial du Pérou (xvil' s., Fondation Abegg (Berne) $n^{\circ}$ 416, d’après Muthmann 1977 pl. I). Noter la ressemblance avec le châle de la figure 6 a.

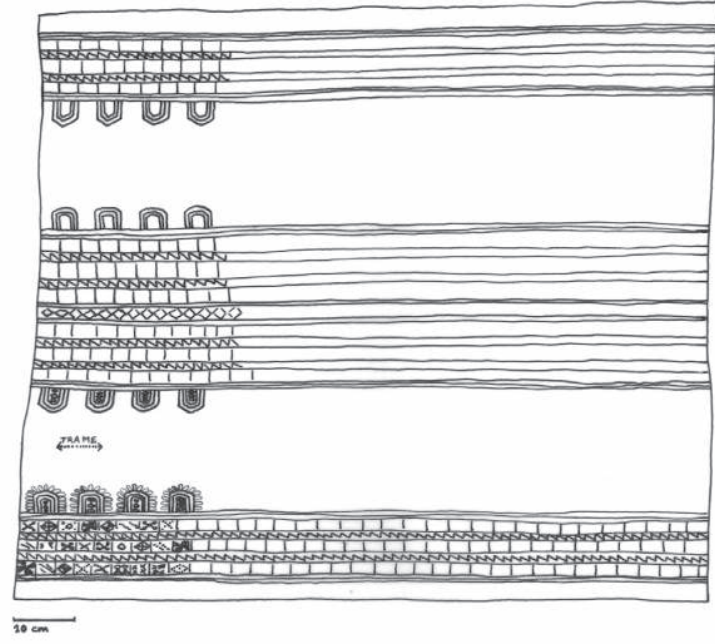

Qu'en est-il pour les pièces de vêtement féminin en tapisserie ? Celles-ci sont extrêmement rares. Deux châles Tiahuanaco provenant du cimetière de San Pedro de Atacama constituent les seuls exemples préhispaniques dont l'analyse ait été publiée. D’après les reconstitutions d'Amy Oakland, ils sont comparables aux tuniques masculines contemporaines : tapisserie à liures simples et lisières longitudinales renforcées, port avec la chaîne horizontale et donc avec l'élément dominant vertical (Oakland 1986 : 104-106). Il n'y a donc pas d'opposition horizontal/vertical entre ces pièces féminines et masculines de Tiahuanaco, mais ce contraste se vérifie dans les pièces postérieures que j’ai pu jusqu'ici identifier. 
Huit châles, et ce qui est probablement une robe de la Période Coloniale comportent un décor avec des motifs de personnages, d'animaux... qui ne laissent pas de doute sur l'orientation de ces étoffes. Et les photos ou descriptions qui les accompagnent sont dans chaque cas suffisamment précises pour quon puisse comprendre que leur trame était alors horizontale - à l'inverse toujours des tuniques masculines de mêmes époque et structure (figure 12) ${ }^{18}$. La plupart présentent un décor comportant des tocapus. Des peintures des XVII et XVIII siècles $^{e}$ et quelques dessins de Guaman Poma ${ }^{19}$ confirment le sens dans lequel les femmes revêtaient ces pièces et semblent montrer que cette opposition existait déjà pour les Incas.

\section{La côte}

Des travaux récents (Kajitani 1982 : fig. 101, Young 1985 : 58, Bruce 1986 : 189-190) apportent une explication tout à fait convaincante au problème posé par la construction inhabituelle d'un nombre réduit de tuniques des côtes septentrionale et centrale du Pérou. Depuis 1955, ces tuniques de la Période Intermédiaire Récente ont provoqué la curiosité des chercheurs (Gay ton 1955, Emery and King 1957, Stumer and Gay ton 1958, VanStan 1961 et 1967, Huepenbecker 1969 : 216-228), car elles sont construites à l'opposé des pratiques habituelles. Les 26 tuniques d'adultes et 11 tuniques miniatures décrites dans la littérature spécialisée ont les ouvertures pour la tête et pour les bras orientées horizontalement. Des 13 exemples dont le contexte archéologique est connu, 12 sont associés à des tombes de femmes, ce qui permet de supposer que ces tuniques étaient féminines. Une pareille idée est renforcée par l'observation selon laquelle les poupées funéraires contemporaines de la culture Chancay qui représentent des personnages féminins semblent revêtues de telles tuniques construites à l'opposé de celles portées par les représentations masculines (figure 13) ${ }^{20}$. Bien qu'elle se soit déplacée sur d'autres traits techniques, l'opposition entre pièces de vêtement féminin et masculin se manifesterait donc aussi sur la côte par un contraste entre les orientations horizontale et verticale.

\section{Le piémont amazonien}

D’après Steward et Métraux (1948 : 543), la même opposition se retrouve dans le piémont amazonien. Chez certains groupes, en particulier de langue arawak et tucano, parfois depuis le $\mathrm{xvl}^{\mathrm{e}}$ siècle, les tuniques portées par les femmes présentent une ouverture pour la tête horizontale tandis que leurs contreparties masculines sont construites avec le même trait orienté verticalement (figures 15 et 16).

Ce dernier exemple est à préciser, comme beaucoup d'autres aspects d'une étude en cours mais suffisamment avancée pour qu'on puisse proposer une idée nouvelle. Il s'agit de reconnaître l'importance des orientations horizontale et verticale dans la lecture technique de certains tissus andins : plus particulièrement les tuniques et ponchos masculins, et les robes, dessus de robe, jupes et peut-être aussi les châles féminins. Les données présentées mettent en parallèle l'opposition horizontal/vertical de divers traits techniques relevés dans ces pièces avec deux schémas d'organisation des sociétés andines : d'une part, une opposition entre la « côte » et les « hautes terres » et d'autre part, le contraste entre le féminin et le masculin.

horizontal : vertical $::$ « côte : hautes terres »

horizontal : vertical :: féminin : masculin 
Bien que la documentation réunie soit très fragmentaire et dispersée, les résultats obtenus forment un tout cohérent. En effet, chaque contraste est relié à d'autres. Tom Zuidema (1964 : 165-170) a montré comment l'opposition « côte/hautes terres » était en relation pour les Incas avec les couples Viracocha/Soleil, terre/ciel, Hurin (bas)/Hanan (haut). Or le dernier contraste est généralement associé dans les Andes à celui entre féminin et masculin (Platt 1976, Harris 1978). On obtient ainsi une série d'oppositions cohérente:

« côte : hautes terres » :: Hurin : Hanan

Hurin : Hanan :: féminin : masculin

Finalement, trop d'éléments concordent pour qu'on puisse penser qu'il s'agit de rapports fortuits. Les oppositions horizontal/vertical observées dans certains tissus des Andes ne résultent pas seulement d'outils et de savoir-faire différents : ils matérialisent quelques-unes des multiples facettes du dualisme andin. Les techniques de tissage ont donc un sens et même un double sens : l'un d'ordre matériel -ordonné par l'entrecroisement des fils de chaîne et de trame - et l'autre relevant du symbolique.

Depuis quand existe ce deuxième sens et dans quelles limites géographiques et ethniques? La documentation rassemblée permet de faire remonter l'opposition « côte/hautes terres » à la fin de la Période Intermédiaire Ancienne ; mais celle entre féminin et masculin se fonde sur des données dont les plus anciennes datent de la Période Intermédiaire Récente. Entre ces extrêmes et aujourd'hui, il est vrai, l'information est lacunaire et peu détaillée. Elle ne permet pas encore de tracer les limites précises de la distinction entre ce que j'ai nommé « côte » et « hautes terres ». Néanmoins, elle suggère la fusion ou la forte relation entre une partie de la côte - la vallée d'Azapa - et les « hautes terres », thème qui intéresse depuis plusieurs années archéologues, ethnohistoriens et linguistes ${ }^{21}$, Elle conduit aussi à établir un lien entre le piémont amazonien et les autres régions, ce qui touche un deuxième domaine de recherche en plein développement (Renard-Casewitz, Saignes, Taylor-Descola 1986).

Des pièces autres que la tunique masculine et le poncho, la robe ou la tunique féminine et peut-être le châle, peuvent présenter des traits techniques où s'opposent horizontale et verticale, par exemple les sacs ou chuspa dont il existe à l'époque préhispanique des modèles presque identiques mais tissés dans des sens opposés, comme l'a illustré A.P. Rowe avec la photo de deux objets provenant probablement de la côte (Rowe 1977 : fig. 58). Des recherches complémentaires permettront peut-être de mieux comprendre ce problème, et éventuellement d'intégrer dans le schéma proposé des pièces non considérées ici : celles qui n’ont pas de décor, celles dans lesquelles la chaîne et la trame contribuent également à former les motifs ou bien d'autres encore, qui sont composées de plusieurs lés dont le décor est crée par des fils orientés selon des directions opposées.

En proposant une nouvelle grille de lecture des tissus andins, cet article devrait contribuer à ce que des informations plus précises sur les textiles apparaissent dans des travaux ethnographiques à venir. Car il semble que si beaucoup de ceux qui ont été à ce jour publiés manquent de précision sur les points ici exposés, c'est moins en raison de la complexité des faits à prendre en compte et à décrire - problème par ailleurs courant dans le domaine des textiles - que de leur banalité qui les rend «invisibles », ou fait juger leur publication superflue. 


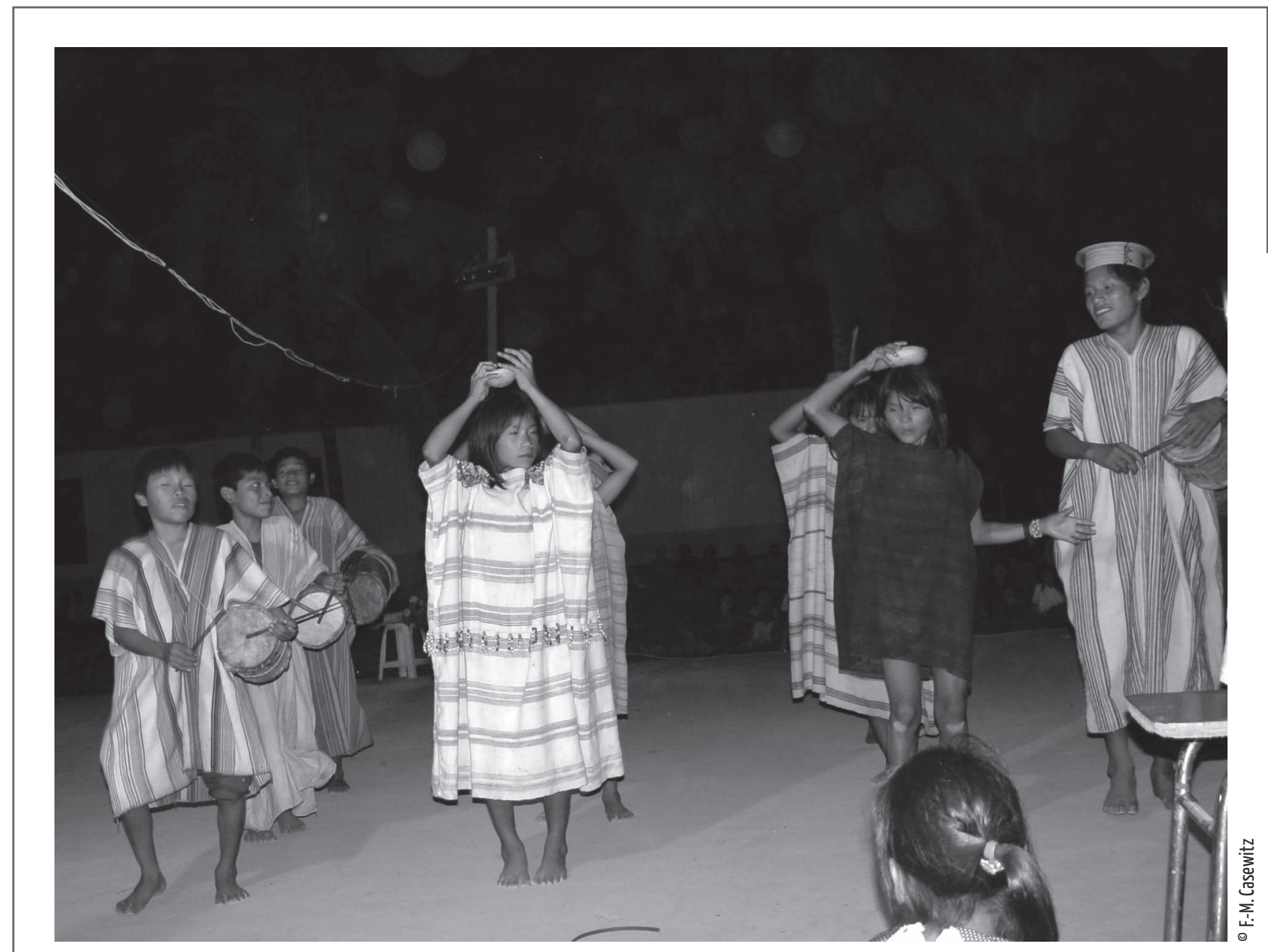

Figure 15

Enfants Matsiguenga lors de la fête du saint de la mission de Kirigueti, département de Cuzco, Pérou, août 2002, (cliché France-Marie Casewitz). 

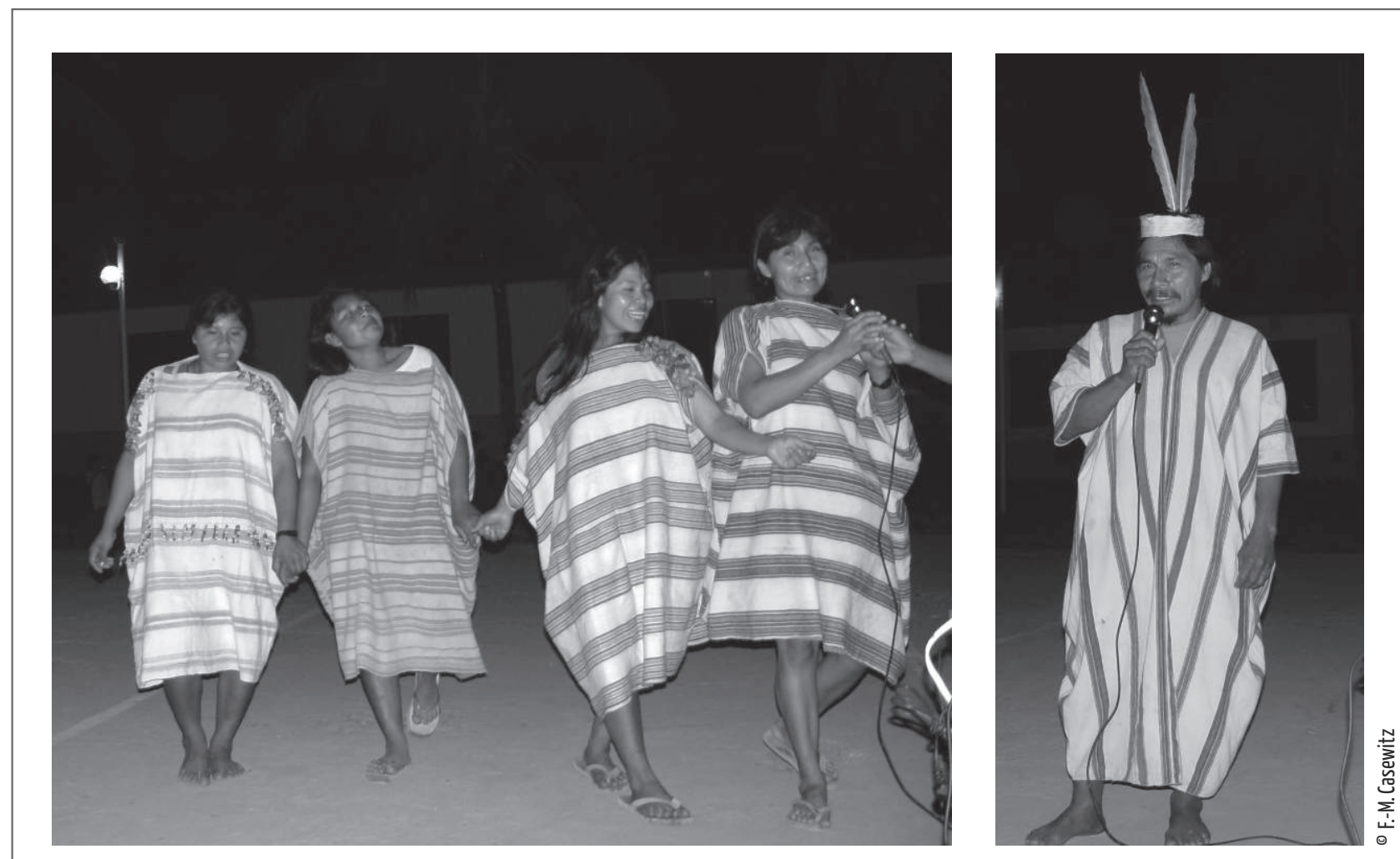

\section{Figure 16. Matsiguenga du Piémont amazonien}

Les ouvertures pour la tête et les bras et les fils de chaîne formant les rayures

sont horizontaux dans les cushma des femmes (a) et verticaux dans celle de l'homme (b).

Kirigueti, département de Cuzco, Pérou, août 2002 (cliché France-Marie Casewitz). 
1. Ce travail reprend une hypothèse formulée dans une thèse soutenue à l'EHESS en juillet 1987. Il a été présenté sous une forme résumée au 46e Congrès International des Américanistes à Amsterdam (juillet 1988). La partie concernant les hautes terres a été exposée à la Reunión anual de Etnología à La Paz (août 1988). De nombreuses personnes, à des titres divers, ont contribué par leurs suggestions à cet article. Je tiens particulièrement à remercier: Thérèse Bouysse-Cassagne, Marie-Noêlle Chamoux, Penny Dransart, Pierre Duviols, Antoinette FioravantiMolinié, Jean-Luc Jamard, Marie-Claude Mahias, Susana Monzón. Thierry Saignes et Nathan Wachtel.

2. Sur ce dernier point, voir par exemple les travaux de Monteil (1929), VanStan (1979), Rowe (1984 :18).

3. Les bibliographies concernant les textiles ethnographiques sud-américains (O'Neale (1949) et SeilerBaldinger (1977)) montrent le désintérêt des ethnologues pour ce sujet pendant le troisième quart de ce siècle. La publication d'un travail similaire pour la dernière décennie mettrait en évidence la reprise actuelle des recherches sur ce thème.

4. «Cordelette d'extrémité » est la traduction la plus satisfaisante que j'ai pu trouver pour le terme anglais heading cord.

5. Tisserand est employé ici comme terme générique, et donc, masculin.

6. La répartition géographique et chronologique de ces diverses variantes n’a pas été récemment analysée en détail, à l'exception des métiers à tension fixe et chaîne verticale étudiés par Vreeland (1979).

7. Les tissus étaient également tissés à quatre lisières et employés sans être coupés en Amérique Centrale (Chamoux et Cousin 1988 : 101).

8. La terminologie quechua employée reprend des formes utilisées par divers auteurs sans chercher à en uniformiser l'orthographe.

9. D’après Monteil (1925), le poncho aurait dérivé de la tunique sous l'influence des Espagnols au Chili à partir du XvII ${ }^{\mathrm{e}}$ siècle. Cette reconstruction a été remise en question par l'existence de pièces semblables déjà à Paracas et plus tard sous la forme de ponchos de plumes pendant la Période Intermédiaire Récente et l'Horizon Récent (King 1965 : 291, Huepenbecker 1969 : 37-52 et 199-207). De même que Rowe (1984 : 175), sans entrer dans celle dispute, je ne considérerai comme poncho que les pièces actuelles qui se différencient des autres par leur qualité et leur fonction.

10. D'Harcourt et d'Harcourt (1924: pl. 40). Sawyer (1963), Huepenbecker (1969 : 275), Bird and Skinner (1974), Rowe (1978), Rowe (1979), Conklin (1985 :
T6 et T7), Oakland (1986). Les lisières longitudinales ne sont pas souvent décrites pour les tuniques Huari mais A. P. Rowe atteste que sur les pièces tissées dans les hautes terres elles sont renforcées (1986 : 153).

11. California Academy of Sciences (1978), Cuesta Domingo (1980 : 105, 170), Kajitani (1982 : pl. 82-83), Conklin (1985 : T1).

12. Bien que de telles tuniques ne soient plus tissées depuis peut-être le début du XIX $x^{e}$ siècle, la tapisserie n'a pas encore été totalement abandonnée dans les Andes. Elle est encore pratiquée au moins à Ayacucho (hautes terres sud) sur des métiers à pédales et à Villa Ribero (département de Cochabamba, Bolivie) sur des métiers verticaux (Gisbert, Arze y Cajías, 1987 : 305-306). Elle l'est peut-être aussi dans la région de Huanuco (hautes terres centrales du Pérou) (Bird and Mendizabal, 1986 : 346). Une enquête effectuée en juillet et août 1988 à Villa Ribero a permis de préciser que la tapisserie y est tissée avec des parfilages simples groupés - ce qui signifie qu'aux changements de couleur, des groupes de trames tournent alternativement autour d'un même fil de chaîne (Viallet 1971 : 42). Des fils de chaîne doubles constituent les lisières longitudinales mais leur nécessité n'a pu être démontrée.

13. Par exemple Guaman Poma (1936 : 92,147, 169, 270, 324, 346, 398, 531, 622, 747, 780), Rowe (1977 fig. 2, 17, 18,70,79, pl 1) Ramlrez del Águila (1978 : face p. 131), Adelson and Tracht (1983: $\mathrm{n}^{\circ} 1-6$, fig. 4, 20, 25, 28), Gisbert, Arze y Cajias (1987 : face p. 60, 180, fig. 28, 32, 39, 43, 67, 68, 99, 266). L'iconographie ne permet pas de savoir avec quelle structure les étoffes représentées ont été tissées. Quand il s'agit de pièces des hautes terres, avec un simple décor de rayures, j'ai supposé qu'elles avaient été exécutées avec des structures créant un décor par la chaîne, la direction des rayures indiquant celle de la chaîne. Quant aux tuniques pré-hispaniques, la plupart sont sans indication de provenance mais le fait qu'elles soient entièrement en poils de camélidés permet de supposer qu'elles ont été fabriquées dans les hautes terres.

14. Ce dernier exemple a probablement été tissé sur la côte en suivant une tradition technique et iconographique originaire des hautes terres. Ceci met en évidence le problème de la distinction entre lieu d'origine et lieu de pratique des techniques qui n'a pu être pris en compte ici.

15. Voir par exemple Adelson and Tracht (1983 : 109, 111, fig. 36) Meisch (1986 : fig. 7.10, 20) Gisbert, Arze y Cajías (1987 : fig. 109, 279, 310).

16. Voir par exemple Girault (1969 : fig. 6,9) Adelson and Tracht (1983 : 111) Gisbert, Arze y Cajías (1987 : 300, 
face fig. $73,78,80,163,180,211$ ) ainsi que Meisch (1986 : fig. 8, 9, 15) Dans les publications, ces châles sont très souvent présentés avec la chaîne verticale sans tenir compte de la manière dont ils sont portés ni du fait que dans les exemples nettement rectangulaires, le plus grand côté devrait être la largeur de la pièce. Ce problème ne se pose pas pour les robes, dessus de robe et jupes qui sont presque uniformément orientés avec la chaîne horizontale.

17. Guaman Poma (1936 : 122, 126, 128, 130, 132, 134 (bas gauche), 138, 173, 223, 242, 258, 318, 377 , 379, 408, 573, 609, 757, 759) Ocaña (1961 (d'après Money, 1983 : verso de page face à 184)), Ramirez del Âguila (1978 : face p. 131), Money (1983 : couverture), Gisbert, Arze y Cajías (1987 : face p. 168, fig. 38, 39, 195). L'iconographie est à nouveau imprécise sur le type de structure employé. Quand il s'agit de pièces des hautes terres, d'une part j'ai distingué les étoffes avec un décor similaire à ceux des tuniques masculines en tapisserie, en particulier celles présentant des tocapus - que j'ai supposées également en tapisserie, et d'autre part j'ai considéré que les étoffes féminines rayées avaient été exécutées avec des structures à dominante chaîne comme leurs contreparties masculines représentées sur les figures 5b et 10 .

18. Zimmern (1943-1944), Muthmann (1977), Kajitani (1982 : pl.125). N. Zimmern (1943-1944: 40) n’a pas classé le n 36.760 du Brooklyn Museum avec les vêtements... mais comme une étoffe liturgique à cause de ses dimensions $(169 \times 139 \mathrm{~cm})$ et de l'asymétrie de son décor. F. Muthmann (1977 : 56 et fig. 30) insiste sur la similarité de cette pièce avec les châles, mais ne l'identifie pas comme robe. C'est pourtant cette hypothèse qui s'impose quand on compare ladite pièce avec d'autres robes ou dessus de robe plus récents tissés à dominante chaîne (Adelson and Tracht, 1983 : nº 39 -40).

19. Rowe (1951 : fig. 4-7), Guaman Poma (1980 : 126, 134, 258, 757). Des châles en tapisserie seraient encore tissés dans les hautes terres centrales et sud du Pérou et, dans un cas, clairement portés avec la trame horizontale (Bird and Mendizabal; 1986 : fig. 24; Bollinger 1983 : face p. 146).

20. Kajitani (1982: fig. 99), Bruce (1986:190); Tsunoyama (1979: pl. 230, 233). J'ai pu constater l'orientation horizontale des ouvertures pour la tête et pour les bras sur deux poupées, sans indication de lieu de provenance du Museo Civico de Modène (Italie): F31 et F33 (fig. 13). Il serait nécessaire d'analyser davantage d'exemples pour s'assurer de la régularité de cette construction.

21. Murra (1976), Mujica, Rivera y Lynch (1983), Mujica (1985), Torero (1987). Il s'agit d'un thème à propos duquel on est loin d'avoir un panorama bien défini si on en juge par le débat qui a suivi l'article de Torero.

\section{RÉFERENCES}

Adelson, L. \& A. Tracht 1983 Aymara Weavings. Ceremonial Textiles of Colonial and 19th Century Bolivia. Smithsonian Institution Traveling Exhibition Service.

Bertonio, P. L. 1984 [1612] Vocabulario de la lengua aymara. La Paz: CERES/IFEA/MUSEF.

Bird, J. B. 1985 The Preceramic Excavations at Huaca Prieta, Chicama Valley, Peru. (J. Hyslop, dir.). With textile sections completed by Milica Dirnitrijevic Skinner (Anthropological Papers of the American Museum of Natural History 62, part I). New York.

Bird, J. B. \& M. D. Skinner 1974 The Technical Features of a Middle Horizon Tapestry Shirt from Peru, Textile Museum Journal 4 (1) : 5-12.

Bird, R. Mck \& L. E. Mendizabal 1986 Textiles, Weaving, and Ethnic Groups of Highland Huanuco, Peru, pp. 339-361, in A.P. Rowe dir. The Junius B. Bird Conference on Andean Textiles. April 7th and 8th, 1984. Washington D. C: The Textile Museum: 339-361.

Bollinger, A. 1983 So kleideten sich die Inka. (Schriftenreihe des Institutes rur Lateinamerikaforschung und Entwicklungszusammenarbeit an der Hochschole St. Gallen 2). Diessenhofen (Suisse) : Rüegger.

Bouysse-Cassagne, T. 1978 L'Espace Aymara: Urco et Uma, Annales. E.S.C. 33(5-6) : 1057-1080.

- 1987 La Identidad Aymara. Aproximación histórica (Siglo xv, Siglo xvi). La Paz: Hisbol/IFEA.

Bruce, S. L. 1986 Textile Miniatures from Pacatnamu, Peru in A. P. Rowe dir. The Junius B. Bird Conference on Andean Textiles. April 7th and 8th, 1984. Washington D. C: The Textile Museum: 183-204. 
California Academy of Sciences 1978 Peruvian Archaeological Textiles. A Weaver's Art. Slide catalog. San Francisco.

Cereceda, V. 1978 Sémiologie des tissus andins: les talegas d'Isluga, Annales E. S. C. 33(5-6): 1017-1035.

Chamoux. M.-N. \& F. Cousin 1988 Héritage culturel et innovation : les blouses des femmes de la Huastèque (Mexique), Techniques \& Culture 11: 95-128.

Conklin. W. J. 1970 Peruvian Textile Fragment from the Beginning of the Middle Horizon, Textile Museum Journal 3 (1) : 15-24.

- 1975 « An Introduction to South American Archaeological Textiles with Emphasis on Material and Techniques of Peruvian Tapestry, in P. L Fiske dir. Irene Emery Roundtable on Museum Textiles, 1974 Proceedings: Archaeological Textiles. Washington D.C. : The Textile Museum: 17-30.

- 1985 Pucara and Tiahuanaco Tapestry: Time and Style in a Sierra Weaving Tradition, Nawpa Pacha 21 (1983): 1-43.

- 1986 The Mythic Geometry of the Ancient Southern Sierra, in A.P. Rowe dir. The Junius B. Bird Conference on Andean Textiles. April 7th and 8th, 1984. Washington D.C. : The Textile Museum: 123-135.

Cuesta Domingo, M. 1980 Arqueología andina: Perú. Madrid: Ministerio de Cultura, Dirección general del patrimonio Artístíco, Archivos y Museos.

Desrosiers, S. 1985 Une Expérience de technologie: la reconstruction d'une ceinture précolombienne à partir d'un texte codé du xvII ${ }^{e}$ siècle, Techniques et culture 6: 111-144.

- 1987 Structures et décors des tissus andins préhispaniques. Thèse de 3e cycle, Ethnologie. Paris: École des Hautes Etudes en Sciences Sociales (multigr.).

Emery, I. \& M.E. King 1957 Additional Examples of an Unusual Peruvian Shirt Type American Antiquity 23 (1): 71-74.

Frame, MP. 1982 Ancient Peruvian Sprang Fabrics. M.A. Thesis, Fine Arts. University of British Columbia (multigr.).

Gayton, A.H. 1955 A New Type of Ancient Peruvian Shirt, American Antiquity 20 (3) : 263-270.

- 1961 The Cultural Significance of Peruvian Textiles: Production, Function, Aesthetics, Kroeber Anthropological Society Papers 25 : 11-128.

Girault, L. 1969 Textiles boliviens. Région de Charazani. (Catalogues du Musée de l'Homme Série H Amérique IV). Paris: Museum National d'Histoire Naturelle.

Gisbert. T. Arze, S. \& M. Cajías 1987 Arte textil y mundo andino. La Paz: Gisbert.

Grieder, T. 1986 Precerarnic and Initial Textiles from La Galgada, Peru, in A. P. Rowe (eds.) The Junius B. Bird Conference on Andean Textiles. April 7th and 8th, 1984. Washington D.C.: The Textile Museum: 19-29.

Guaman Poma De Ayala, F. 1936 [1613?] Nueva Coronica y Buen Gobierno. Paris: Institut d'Ethnologie.

D'Harcourt, R. 1934 Les Textiles anciens du Pérou et leurs techniques. Paris: Les Éditions d'Art et d'Histoire.

D’Harcourt, M. \& D'Harcourt 1924 Les Tissus indiens du vieux Pérou. Paris: A. Morancé.

Harris, O. 1978 Complementary and Conflict: an Andean View of Women and Men, in J. La Fontaine (eds.) Sex and Age as a Princip le of Social Differenciation. New York: Academic Press.

Huepenbecker, A. L. 1969 Pre-Columbian Peruvian Costume: Characteristics and Variations in the PonchoShirt. Ph.D. dissertation. Ohio State University (multigr.).

Kajitani, N. 1982 Andesu No Senshoku (Textiles of the Andes), Senshoku no bi (Textile Art) 20 : 10-96.

King, M. E. 1965 Textiles and Basketry of the Paracas Period, Ica Valley, Peru. Ph. D. dissertation, Anthropology. University of Arizona (multigr.).

Lothrop, S. K. 1979 Les Trésors de L’Amérique précolombienne. Genève: Skira/Flammarion.

Lumbreras, L. G. 1974 The Peoples and Cultures of Ancient Peru. Washington D.C. : Smithsonian Institution Press.

March, K. S. 1983 Weaving, Writing and Gender, Man 18: 729-744.

Meisch, L. A. 1986 Weaving Styles in Tarabuco, Bolivia, in A.P. Rowe dir. The Junius B. Bird Conference on Andean Textiles. April 7th and 8th, 1984. Washington D.C.: The Textile Museum: 243-274.

Messick, B. 1987 Subordinate Discourse: Women, Weaving, and Gender Relations in North Africa, American Ethnologist 14 (2) : 210-225. 
Métraux, A. 1934-1935 La Survivance du vêtement incasique chez les Indiens Chipaya de Carangas, Bulletin du Musée d'Ethnographie 8 : 15-24.

Money, M. 1983 Los Obrajes, el traje y el comercio de ropa en la audiencia de Charcas. La Paz: Instituto de Estudios Bolivianos/Facultad de Humanidades UMSA.

Montell, G. 1925 Le Vrai poncho, son origine postcolombienne, Journal de la Société des Américanistes 17: 173183.

- 1929 Dress and Ornaments in Ancient Peru. Londres: Oxford University Press.

Mujica, E. 1985 Altiplano-Coast Relationships in the South-Central Andes: From Indirect to Direct Complementarity, in S. Masuda, I. Shimada \& C. Morris dir. Andean Ecology and Civilization. Tokyo: University of Tokyo Press.

Mujica, E. Rivera, M. A. \& T.F. Lynch 1983 Proyecto de estudio sobre la complementaridad económica Tiwanaku en los valles occidentales del centro-sur andino, Revista Chúngara 11: 85-109.

Murra, J. V. 1962 Cloth and its Functions in the Inca State, American Anthropologist 64(4) : 710-728.

- 1976 « El control vertical de un maximo de pisos ecológicos en la economía de las sociedades andinas, in Formaciones Económicas y Políticas del Mundo Andino. Lima: Instituto de Estudios Peruanos: 59-115.

Muthmann, F. 1977 Eine peruanische Wirkerei der spanischen Kolonialzeit. (Monographien der Abegg-Stiftung Bern 13). Berne: Abegg Stiftung.

Niessen, S. A. 1985 Motifs of Life in Toba Batak Texts and Textiles. (Verhandelingen van het Koninklijl Instituut voor Taal-, Land-en Volkenkunde 110). Dordrecht (Holland)/Cinnaminson (USA): Foris Publications.

Oakland, A. 1986 Tiahuanaco Tapestry Tunics and Mantles from San Pedro de Atacama, Chile in A. P. Rowe dir. The Junius B. Bird Conference on Andean Textiles. April 7th and 8th, 1984. Washington D. C: The Textile Museum: 101-121.

Ocaña, Fray Diego de 1961 [1613 ?] Un viaje fascinante por la América Hispana. Madrid: Studium.

O'Neale, L. M. 1949 Basketry and Weaving in J.H. Steward dir. Handbook of South American Indians 5. (Smithsonian Institution, Bureau of American Ethnology, Bulletin 143). Washington D. C: U.S. Government Printing Office: 69-138.

Platt, T. 1976 Espejos y Maiz: temas de la estructura simbólica andina. La Paz: Cuademos CIPCA.

Ramirez del Aguila, L. P. 1978 [1639] Noticias Politicas de Indias Y Relación Descriptiva de la Ciudad de la Plata Metrópoli de las Provincias de Charcas y Nuevo Reyno de Toledo en las Occidentales dei gran Imperio del Pirú. Transcripción de Jaime Urioste Arana. Sucre: Imprenta Universitaria.

Renard-Casewitz, F.-M., Saignes, T. \& A.-C. Taylor-Descola 1986 L'Inca, l'Espagnol et les sauvages. Rapports entre les sociétés amazoniennes et andines $d u X^{e}$ au XvII siècle. Paris: Éditions Recherches sur les Civilisations (Synthèse $n^{\circ} 21$ ).

Rivera, M. A. 1984 Acerca de la arqueología andina del norte de Chile: Comentarios y referencias bibliográficas publicadas entre 1980-1983, Revista Andina 1: 283-298.

Rowe, A. P. 1977 Warp-Patterned Weaves of the Andes. Washington D.C. : The Textile Museum.

- 1978 Technical Features of Inca Tapestry Tunies, Textile Museum Journal 17 : 5-28.

- 1984 Costumes and Featherwork of the Lords of Chimor. Washington D.C. : The Textile Museum.

- 1986 Textiles from the Nasca Valley at the Time of the Fall of the Huari Empire, in A. P. Rowe dir. The Junius B. Bird Conference on Andean Textiles. April 7th and 8th, 1984. Washington D.C.: The Textile Museum: 151-182.

Rowe, J. H. 1951 Colonial Portraits of Inca Nobles, in Sol Tax (eds.) The Civilizations of Ancient America: Selected Papers of the XXIXth International Congress of the Americanists vol. 1. Chicago: University of Chicago Press: 258-268.

- 1979 Standardization in Inca tapestry tunic in A. P. Rowe, E. P. Benson \& A.-L. Schaffer dir. The Junius B. Bird pre-Columbian Textile Conference. Washington D.C.: The Textile Museum: 239-264.

Rowe, J.H. \& D. Menzel dir. 1982 [1967] Peruvian Archaeology. Selected Readings. Palo Alto: Peek Publications.

Sawyer, A. R. 1963 Tiahuanaco Tapestry Design, Textile Museum Journal 1 (2) : 27-38. 
Schmidt, M. 1910 Szenenhaften Darstellungen auf altperuanischen Gewebe, Zeitschrift for Ethnologie 42: 154164.

- 1911 Über altperuanische Gewebe mit szenenhaften Darstellungen, Baessler Archiv 1: 1-61.

Seiler-Baldinger, A. 1977 General Introduction to the Literature on South American Ethnographic Textiles since 1950, in I. Emery \& P. Fiske dir. Irene Emery Roundtable on Museum Textiles. 1976 Proceedings: Ethnographie Textiles of the Western Hemisphere. Washington D.C.: The Textile Museum.

Steward, J. H. \& A. Métraux 1948 Tribes of the Peruvian and Ecuadorian Montaña, in J.H. Steward dir. Handbook of South American Indians 3. (Smithsonian Institution, Bureau of American Ethnology, Bulletin 143). Washington D. C: U.S. Government Printing Office: 535-656.

Stumer, L. M. \& A. H. Gayton 1958 A Horizontal-Necked Shirt from Marques, Peru, American Antiquity 24 (2): 181-182.

Taullard, A. 1949 Tejidos y ponchos indígenas de Sudamérica. Buenos Aires: Editorial Guillermo Kraft.

Torero, A. 1987 Lenguas y pueblos altiplánicos en torno al siglo XVI, Revista Andina 11 : 329-405.

Torrico, C. 1988 Living Weavings: the Symbolism of Bolivian Herders Saeks (texte manuscrit.)

Tsunoyama, Y. 1979 Textiles of the Andes. Catalog of Amano Collection. San Francisco: Heian International.

Uhle, M. 1903 Pachacamac. Report of the William Pepper, M.D., LL.D., Peruvian Expedition of 1896. Philadelphia: University of Pennsylvania, Department of Archaeology.

Ulloa, T. L. 1981a Evolución de la industria textil prehispánica en la zona de Arica, Revista Chúngara 8: 97108.

- 1981b Estilos decorativos y formas textiles de poblaeiones agromarítimas en el extremo norte de Chile, Revista Chúngara 8: 109-136.

Vanstan, I. 1961 Miniature Peruvian Shirts with Horizontal Neck Openings, American Antiquity 26 (4) : 524531.

- 1967 Textiles from Beneath the Temple of Pachacamac, Peru: A Part of the Uhle Collection of the University Museum, University of Pennsylvania. Philadelphia: University Museum

- 1979 Did Inca Weavers Use an Upright loom? in A.P. Rowe, E. P. Benson \& A.-L. Schaffer dir. The Junius B. Bird pre-Columbian Textile Conference. Washington D.C.: The Textile Museum/Dumbarton Oaks: 233238.

Viallet, N. 1971 Tapisserie. Principes d'analyse scientifique. Paris: Ministère des Affaires Culturelles/Inventaire Général des Monuments et des Richesses Artistiques de la France.

Vreeland, I. M. Jr 1979 The Vertical Loom in the Andes, Past and Present, in I. Emery \& P. Fiske dir. Irene Emery Roundtable on Museum Textiles: 1977 Proceedings: Looms and their Products. Washington D.C.: The Textile Museum: 188-211.

Wallace, D. T. 1979 The Process of Weaving Development on the Peruvian Coast, in A. P. Rowe, E. P. Benson \& A.-L. Schaffer dir. The Junius B. Bird pre-Columbian Textile Conference. Washington D.C. : The Textile Museum/Dumbarton Oaks: 27-50.

Young, M. A. 1985 The Chancay textile tradition: textiles from Lauri in the Collection of the Amano Museum. M.A. Thesis. Columbia University (multigr.).

Zimmern, N. 1943-1944 The Tapestries of Colonial Peru, Brooklyn Museum Journal: 27-52.

Zorn, E. 1986 Textiles and Herder's Ritual Bundles of Macusani, Peru in A. P. Rowe dir. The Junius B. Bird Conference on Andean Textiles. April 7th and 8th. 1984. Washington D.C. : The Textile Museum: 89-307.

- 1987 Un análisis de los tejidos en los atados rituales de los pastores, Revista Andina 5(2): 489-526.

Zuidema, R.T. 1964 The Ceque System of Cuzco. The social Organization of the Capital of the Inca. Leiden: E. J. Brill. 


\section{RÉSUMÉ}

Les techniques de tissage ont-elles un sens? Un mode de lecture des tissus andins. L'analyse comparative de l'histoire des techniques textiles dans les Andes Centrales et Centre-Sud témoignent de la présence d'oppositions stylistiques de nature technique. Depuis la fin de la Période Intermédiaire Ancienne, des jeux d'opposition sont matérialisés dans l'orientation horizontale ou verticale de la chaîne, de la trame ou d'autres caractéristiques techniques de certaines pièces de vêtements. Ces contraintes coïncident avec une division de l'espace en deux grandes régions géographiques - la « côte » et les « hautes terres»- et, localement, avec une distinction entre le féminin et le masculin. De telles observations conduisent à faire l'hypothèse que, depuis plusieurs siècles, les différentes techniques intégrées dans ces tissus correspondent à des distinctions importantes entre catégories sociales dans le monde andin.

\section{ABSTRACT}

Are Weaving Techniques One Way Street? A Way of Looking at Andean Textiles. Comparative analysis of the history of textiles from the Central and Central-Southern Andes suggests that certain basic stylistic polarities arise from technical distinctions. Since the end of the Early Intermediate Period, polar contrasts based on the horizontal or vertical orientation of the warp, the weft, or of certain other technical features, show up in particular items of clothing. These polarities are superimposed upon a spatial division into two geographical subregions corresponding approximately to the coast-highland distinction and, locally, upon a female-male opposition. Such observations lead to the hypothesis that, over centuries, the technical distinctions embodied in these textiles were made to fit with important social categories within the Andean world. 


\section{NOTES ET RÉF́ERENCES DE L'ITRODUCTION}

En ouverture : poupées funéraires Chancay. Personnages féminin et masculin vêtus d'une tunique avec les ouvertures destinées à la tête et aux bras, orientées horizontalement pour la première et verticalement pour le second (Museo civico arqueologico etnologico di Modena, Italie, $n^{\circ}$ d'inventaire F 33 et F 34). Ces images remplacent la figure 14 de l'article originel. Les figures 15 et 16 ont été ajoutées à l'occasion de cette réédition.

L'auteur tient particulièrement à remercier la directrice du Museo civico arqueologico etnologico di Modena, Dra. Ilaria Pulini, pour les photos des poupées funéraires Chancay (en ouverture) et France-Marie Casewitz pour lui avoir permis d'étudier et de reproduire les clichés pris à la fête de la mission de Kirigueti (figures 15 et 16).

Sauf mention contraire toutes les illustrations sont la propriété de l'auteur.

1. Voir par exemple Delmarcel 1999, p. 12.

2. Sauf cas d'artiste cartonnier créant une composition avec une majorité de lignes horizontales mieux rendues en commençant le tissage par le bas (voir par exemple Verlet et al. 1977, p. 206).

3. Malgré ce que les titres de certaines publications voudraient faire croire [Writing with Thread].

4. La remarque d'A. Leroi-Gourhan 1971, [p. 272-273 « la primauté de l'enchevêtrement sur le moyen de l'obtenir est telle qu'on ne peut généralement pas dire, sur examen d'une étoffe, quel métier a servi à la fabriquer... »] ne reflète que son intérêt limité pour les textiles. Il arrive très souvent que les entrelacements de fils dont sont constitués les tissus gardent des traces des métiers employés pour les entrelacer.

5. Voir par exemple Rowe 1990-1991 et 2001-2002, Horié 1990-1991, Frame 1997-1998 et 2003-2004, Rodman et Fernandez 2005 (p. 125), ainsi que Boytner 2004 dont la critique, curieusement, ne tient pas compte des observations ethnographiques présentées dans l'article.

Boytner, R. 2004 Clothing the Social World, p. 130-145, in H. Silverman (dir), Andean Archaeology, Oxford : Blackwell.

Delmarcel, G. 1999 La Tapisserie flamande du XV au XvIII siècle, Paris : Imprimerie nationale.

Desrosiers, S. 1992 Las Tecnicas de tejido itienen un sentido? Una propuesta de lectura de los tejidos andinos, Revista Andina 10 (1) : 7-46.

Frame, M. 1997-1998 Chuquibamba: A Highland Textile Style, Textile Museum Journal 36-37 : 3-47.

- 2003-2004 What the Women Were Weaving: A Deposit of Early Nasca Dresses and Schawls from Cahuachi, Peru, Textile Museum Journal 42-43: 13-53.

Horié, Donna M. 1990-1991 A Family of Nasca Figures, Textile Museum Journal 29-30 : 77-92.

Leroi-Gourhan, A. 1971. L’Homme et la Matière. Paris : Albin Michel (1e édition 1943).

Rodman, A. O. et G. A Fernandez L. 2005 North Coast Style after Moche: Clothing and Identity at El Brujo, Chicama Valley, Peru : 115-133, in R. M. Reycraft (ed), Us and Them: Archaeology and Ethnicity in the Andes, Los Angeles: Cotsen Institute of Archaeology, University of California.

Rowe, Ann P. 1990-1991 Nasca Figurines and Costume, Textile Museum Journal 29-30 : 93-128.

— 2001-2002 Inca style Woman's Dress, Textile Museum Journal 40-41 : 99-117.

Verlet, P., Florisoone M., Hoffmeister A. et Tabard F. 1977 La Tapisserie. Histoire et technique du XIve au XXe siècle, Paris: Hachette.

Writing with Thread. Traditional Textiles of Southwest Chinese Minorities. Catalogue d'exposition, Honolulu : Université d'Hawai'i Art Gallery, 2009. 